\title{
OXIDATIVE BURST OF KUPFFER CELLS: TARGET FOR LIVER INJURY TREATMENT
}

\author{
Jiří Vrba, Martin Modrianský \\ Institute of Medical Chemistry and Biochemistry, Faculty of Medicine, Palacký University, Hněvotínská 3, \\ 77515 Olomouc, Czech Republic
}

Received: September 15, 2002

Key words: Oxidative stress / Macrophages / Natural substances / Inflammation

Liver injury, and frequent consequent fibrosis, is the focus of a number of research groups ranging from molecular biologists to clinicians. It encompasses many aspects and approaches. Among biochemical events the role of Kupffer cells, oxidative stress and pro-inflammatory mediators are eminent. In this review we focus on recent findings into use of natural substances for the modulation of oxidative burst as well as production of inflammatory mediators by Kupffer cells.

\section{INTRODUCTION}

Extensive liver injury of various aetiology, manifested as hepatocyte damage and associated with inflammatory and immune responses, is the starting point for events which may result in fibrosis, often followed by cirrhosis. Liver inflammation is initiated by Kupffer cells, the resident macrophages of the liver, and it involves the formation and release of many inflammatory mediators thereby recruiting neutrophils, lymphocytes, and other inflammatory cells into damaged regions ${ }^{1}$. It is a complex and multifactorial process in which hepatocytes, other nonparenchymal cells, and infiltrating inflammatory cells interact with one another through soluble mediators, surface receptors, and adhesion molecule expression ${ }^{2}$.

The largest organ in the human body, the liver consists of parenchymal cells, i.e. hepatocytes which constitute approximately $80 \%$ of total cell number, and nonparenchymal cells of the sinusoids: Kupffer cells, endothelial cells, hepatic stellate cells (HSC) and Pit cells ${ }^{3}$.

The majority of Kupffer cells are found in the liver periportal regions either in the gaps between adjacent sinusoidal endothelial cells or on their surface ${ }^{4}$. They are larger than endothelial cells and have an irregular stellate shape. Their cytoplasm is rich in organelles and projects in the form of pseudopodia and microvilli into the lumina of sinusoids. The cytoplasm contains phagocytic vesicles, which in turn can contain cellular fragments and haemosiderin coming from phagocytosis and destruction of old erythrocytes 5 . A notable feature of Kupffer cells are worm-like tubules located in their cytoplasm probably representing reservoirs of cell membrane available for rapid phagocytic response to particulate matter ${ }^{6}$. Although all macrophages are ultimately derived from the stem cells of the bone marrow, Kupffer cells can also propagate in the liver sinusoids. In the rat liver Kupffer cells represent about $15 \%$ by cell number and $3 \%$ by mass ${ }^{7}$.

\section{KUPFFER CELL FUNCTIONS}

Kupffer cells, as well as other macrophages, are unique cells for their role in both the innate and acquired immunity. The primary functions of Kupffer cells are phagocytosis, processing of ingested material, antigen presentation and secretion of biologically active products $^{2}$. For these purposes they contain receptors on their surface and binding sites in the cytosol for specific ligands of phagocytosable particles and for many soluble substances (Table 1).

The contact between receptor and a respective particle can activate or stimulate Kupffer cells. Full macrophage activation is a two-stage process, which requires a cytokine, e.g. interferon- $\gamma($ IFN- $\gamma$ ) and an elicitor like lipopolysaccharide (LPS $)^{7}$ (Fig 1). In this model IFN- $\gamma$ primes macrophages and the elicitor converts them to the fully activated form. Primed macrophages are characterised by increased secretion of reactive oxygen species (ROS), expression of Class II major histocompatibility (MHC) molecules and lymphocyte function-associated antigen-1 molecules, and by decreased expression of the transferrin receptor. These cells also have the capacity to bind tumour cells in the absence of antibody. Primed macrophages are activated for presentation of antigen to $\mathrm{T}$ lymphocytes. Fully activated macrophages are characterised by intense secretion of cytolytic proteinase and tumour necrosis factor $\alpha(\mathrm{TNF}-\alpha)$, and are activated for the destruction of tumour cells. Their capacity for presentation of antigen 
Table 1. Ligands and activators of Kupffer cells

\begin{tabular}{|l|l|}
\hline \multicolumn{1}{|c|}{ Ligands } & \multicolumn{1}{c|}{ Activators } \\
\hline$F_{\mathrm{c}}$ part of immunoglobulins & IFN- $\gamma$ (macrophage-activating factor) \\
\hline mannose/N-acetylglucosamine & LPS (endotoxin) \\
\hline fucose & TNF $\alpha$ (cachectin) \\
\hline galactose/N-acetylgalactosamine & virus (Sendai, Newcastle disease) \\
\hline platelet-activating factor & platelet-activating factor \\
\hline apolipoprotein C (high density lipoproteins) & muramyl dipeptide \\
\hline scavenger receptor for modified lipoproteins & nucleotide triphosphates and diphosphates \\
\hline complement & PMA \\
\hline cytokines: IL-1, -4, -6, -10, IFN, TNF, TGF- $\beta$ & ionophores $\left(\mathrm{H}^{+}\right.$and $\left.\mathrm{Ca}^{2+}\right)$ \\
\hline hormones: insulin, prostaglandins & \\
\hline
\end{tabular}

List of substances is based on Refs. ${ }^{2,7}$.

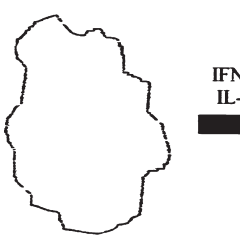

Responsive macrophages express TFR

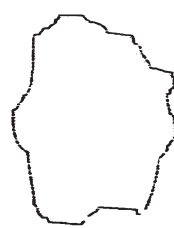

Primed macrophages express Class II
LFA-1 secrete ROS

bind tumour cells present antigen microbicidal action

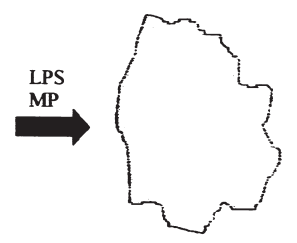

Activated macrophages express Class II MHC LFA-1 secrete ROS TNF- $\alpha$ bind tumour cells kill tumour cells microbicidal action

Fig. 1. Macrophage activation steps. Activation of macrophages may be one or two step process elicited by ligands or activators noted above arrows. MP, maleylated proteins; LFA-1, lympocyte function-associated antigen-1 molecule; TFA, transferrin receptor. Adapted from ${ }^{8}$.

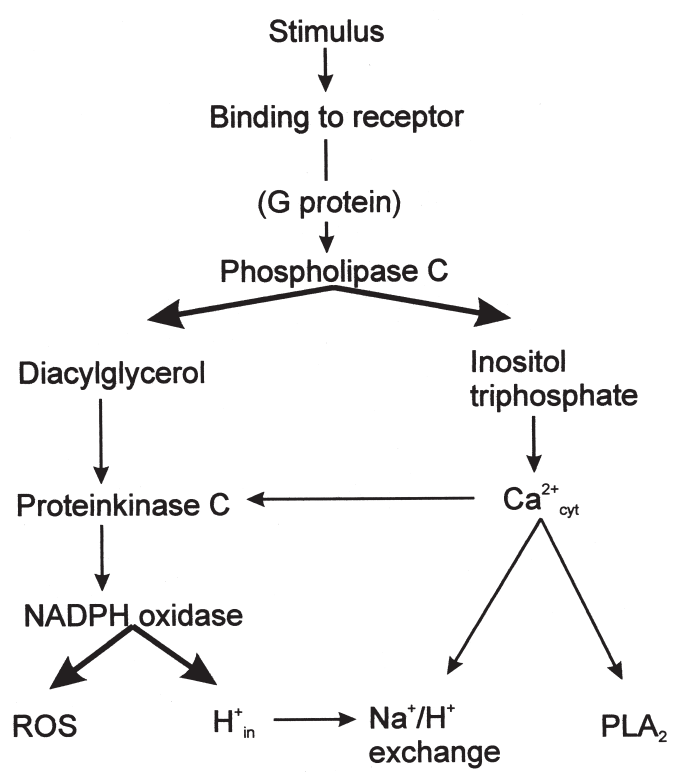

Fig. 2. Signalling pathway for oxidative burst. Thin arrows represent stimulatory actions, thick arrows represent reactions mediated by particular enzyme. $\mathrm{Ca}^{2+}{ }_{\text {cyt }}$, cytosolic free calcium; ROS, reactive oxygen species; $\mathrm{H}^{+}{ }_{\text {in }}$, intracellular proton; PLA $_{2}$, phospholipase $A_{2}$. Adapted from ${ }^{7}$.

Table 2. Biologically active substances produced by Kupffer cells:

- Cytokines:

TNF- $\alpha$, TGF- $\alpha$, TGF- $\beta$, IFN- $\alpha$, IFN- $\beta^{2}$

IL- $1 \alpha$, IL- $1 \beta^{10}$

IL-6, IL-10, CSFs, PDGF 2

MIP-2 29

- Lipid substances:

a) Platelet-activating factor

b) Derivatives of arachidonic acid (eicosanoids):

prostanoids: prostacyclin, prostaglandins (D2, E2, F2), thromboxane A2

leukotrienes: 5-HETE, leukotrienes (B4, C4, D4)

- Inorganic compounds:

a) Reactive oxygen species

b) Nitric oxide?

CSF, colony-stimulating factor; PDGF, platelet-derived growth factor; MIP, macrophage-inflammatory protein; 5-HETE, 5-hydroxyeicosatetraenoic acid

is reduced in comparison with primed macrophages ${ }^{8}$. Interspecies differencies exist for Kupffer cell behavior towards activators. For example mouse Kupffer cells require a two-stage activation in order to become cytotoxic and to release eicosanoids, whereas rat Kupffer cells are in the primed state already and need an elicitor such as LPS or muramyl dipeptide only?

Activated Kupffer cells produce several groups of biologically active substances (Table 2), which confer many functions including clearance and destruction of bacteria, yeast, parasites, endotoxins, tumour cells and particular cell debris, defence against viruses, modulation of immune and inflammatory responses, tissue and matrix remodelling, control of hepatocyte functions, metabolism of iron and bilirubin, and regulation of haematopoiesis and clotting 2 .

Specific ligand-receptor interaction may be transduced to the site of realization in some cases by GTPbinding proteins (Fig. 2). These proteins activate phospholipase $\mathrm{C}^{9}$ and adenylate cyclase ${ }^{7}$. Phospholipase $\mathrm{C}$ hydrolyzes phosphatidylinositol to inositol-1,4,5-triphosphate (InsP3) and diacylglycerol. The former can mediate calcium ion mobilization from the endoplasmic reticulum while the latter activates cytosolic protein kinase $\mathrm{C}$ (PKC) with translocation to the membrane ${ }^{9}$. Experiments with 
rat Kupffer cells indicate that $\mathrm{PKC}$ is involved in the activation of the $\mathrm{Na}^{+} / \mathrm{H}^{+}$antiporter and the NADPH oxidase leading to oxidative burst whereas $\mathrm{Ca}^{2+}$ influx is necessary for phospholipase A activation and eicosanoid synthesis. The formation of cyclic AMP leads to collagenase synthesis and release by rat Kupffer cells ${ }^{7}$, and to activation of protein kinase $\mathrm{A}$ and tyrosine kinase ${ }^{9}$.

\section{Pro-inflammatory cytokines produced by Kupffer cells and modulation of their expression/activity}

The main pro-inflammatory cytokines released by Kupffer cells in response to hepatocellular stress, interleukin-1 (IL-1), interleukin-6 (IL-6), and TNF- $\alpha$, stimulate hepatocytes and other nonparenchymal cells in a paracrine manner. Stimulated sinusoidal endothelial cells express cell adhesion molecules such as intercellular adhesion molecule-1 (ICAM-1) and mediate immigration of neutrophils and blood monocytes supported by chemokines released by HSCs. Deteriorated hepatocytes may then be removed by mononuclear phagocytes $^{10}$.

Various compounds may inhibit the formation of pro-inflammatory cytokines thus offering a means for modulation of the process and possibly avoiding extensive damage (inflammation) of the surrounding tissue by invading neutrophils and lymphocytes. TNF- $\alpha$ release from LPS-stimulated rat Kupffer cells requires internalization of LPS, acidification of endosomes, elevation of intracellular calcium, PKC activation, and protein tyrosine kinase activation. Thus, TNF- $\alpha$ production in rat Kupffer cells may be suppressed by: i) inhibitors of internalization such as cytochalasin B and monodansylcadaverine; ii) inhibitors of endosomal acidification such as bafilomycin $\mathrm{A}$ and monensin; iii) calcium channel blocker nisoldipine; iv) PKC inhibitor $\mathrm{H}-7^{11}$; and v) protein tyrosine kinase inhibitors tyrphostin ${ }^{11,12}$, lavendustin, and genistein. Tyrphostin also inhibits phagocytosis of latex beads by LPS-stimulated rat Kupffer cells in vivo ${ }^{12}$. Broad spectrum protein kinase inhibitors, staurosporine and $\mathrm{K} 252 \mathrm{a}$, and PKC specific inhibitors, calphostin Cand chelerythrine, diminish TNF- $\alpha$ production in human peripheral blood monocytes stimulated with either LPS or zymosan. These data suggest that an initial step in TNF$\alpha$ production in human monocytes challenged with LPS or zymosan involves a PKC dependent mechanism ${ }^{13}$. Amaryllidaceae alkaloids, lycorine and lycoricidinol, inhibit TNF- $\alpha$ production in murine macrophages stimulated in vitro with LPS or treated by Gram-positive bacteria Enterococcus faecalis. Although the inhibition of TNF- $\alpha$ production by lycoricidinol was mainly due to the inhibition of protein biosynthesis, lycorine showed inhibition against TNF- $\alpha$ production at lower concentrations than in the case of inhibition of cysteine/ methionine incorporation into macrophages. It suggests the inhibition of TNF- $\alpha$ production is not due to the inhibitory activity against protein translation, at least at lower concentrations ${ }^{14}$. Biscoclaurine alkaloid cepharanthine suppresses the production of TNF- $\alpha$, IL-1 $\beta$, and IL-8 in human monocytic cell line U937 stimulated with phorbol myristylacetate (PMA) and in human primary monocyte/macrophage cultures stimulated with LPS. In the latter case cepharanthine also suppresses the production of IL- $6^{15}$. Microtubule disrupting agent colchicine and its derivative, colcemide, reduce production of IFN- $\gamma$ in human peripheral blood mononuclear cells with and without prestimulation by LPS ${ }^{16}$. Pretreatment with colchicine inhibits LPS-induced production of granulocyte-macrophage colony-stimulating factor (GM-CSF) and IL-6, but does not affect LPSstimulated induction of inducible nitric oxide synthase in the murine macrophage cell line RAW 264. Studies using stable transfectants reveal that colchicine impairs the transcriptional responsiveness of a reporter gene driven by a GM-CSF promoter sequence. Colchicine inhibition of the GM-CSF response correlates with decrease in the mRNA levels of $\beta$-tubulin. Microtubule agents, colchicine, nocodazole, and podophyllotoxin, inhibit LPS induction of TNF- $\alpha$ in RAW 264 cells. LPS stimulation of macrophages down-regulates levels of $ß$ tubulin transcripts. The ability of both colchicine and LPS to modulate transcription of $\beta$-tubulin suggests that this event does not underlie the inhibitory effect of colchicine on LPS-induced GM-CSF expression. Colchicine probably inhibits LPS induction of GM-CSF by affecting microtubule-dependent costimulatory signaling pathways that synergize with primary LPS-triggered responses ${ }^{17}$.

\section{Oxidative burst in Kupffer cells}

ROS include superoxide anion $\left(\mathrm{O}_{2}^{-}\right)$, hydroxyl radical (HO), and their by-products (e.g., hydrogen peroxide $\left.\mathrm{H}_{2} \mathrm{O}_{2}\right)^{18}$. They do not act in specific ways through receptors or binding sites rather they exploit their chemical reactivity to any suitable compounds with which they collide ${ }^{7}$. ROS are capable of causing oxidative damage to macromolecules encompassing lipid peroxidation, oxidation of amino acid side chains, formation of protein-protein cross-links, oxidation of polypeptide backbones resulting in protein fragmentation, DNA damage, and DNA strand breaks ${ }^{18}$.

Hydroxyl radical is probably the most reactive and cytotoxic oxygen radical, but has a very short half-life of $10^{-9}$ seconds and can diffuse less than $2 \mathrm{~nm}$ from the site of the formation. $\mathrm{H}_{2} \mathrm{O}_{2}$ and superoxide are less reactive and have a longer half-life. Only $\mathrm{H}_{2} \mathrm{O}_{2}$ can easily diffuse across plasma membrane and into the cell ${ }^{19}$.

Thus, oxidative burst, i.e. the rapid uptake of molecular oxygen and its transformation into ROS, represents both host defence mechanisms at the site of injury and the possibility of damage for adjacent healthy tissue ${ }^{1}$. It is an early event in Kupffer cell activity: increased ROS production in response to a stimulus takes place within seconds while increased production of cytokines and other factors takes several hours. Also, the respiratory burst itself, and the accompanying oxidative stress, is one of the stimuli involved in increased expression of cytokines. 


\section{Oxidative burst and hepatitis}

Small amounts of ROS, produced as a consequence of electron transfer reactions in mitochondria, peroxisomes, and cytosol, are scavanged by the cellular defence system including enzymatic antioxidants (e.g., catalase, superoxide dismutase, aldehyde dehydrogenase, GSSG reductase) and non-enzymatic antioxidants (e.g., $\alpha$-tocopherol, glutathione, $\beta$-carotene, bilirubin $)^{18,19}$. High doses of ROS, often generated during acute and chronic inflammatory diseases, are cytotoxic ${ }^{18}$. The term oxidative stress refers to the outcome of oxidative damage to biologically relevant macromolecules such as nucleic acids, proteins, lipids and carbohydrates. Oxidative stress occurs when oxidative stress-related molecules, generated in the extracellular environment or within the cell, exceed intracellular antioxidant defences. Most of the hepatic antioxidant defences are essentially confined to parenchymal cells. Kupffer cells, HSCs, and endothelial cells are potentially more exposed or sensitive to oxidative stress-related molecules. Oxidative stress represents a key feature of hepatitis induced by various conditions, including anoxic/reoxygenation injury, autoimmune hepatitis, viral hepatitis and alcoholic hepatitis. Activated neutrophils, macrophages and Kupffer cells, are a major source of ROS during inflammation. If oxidative stress is severe, all the major cellular structures (particularly mitochondria and cytoskeletal proteins), macromolecules (DNA, lipids, enzymatic proteins) and metabolic pathways can be oxidized, damaged and then blocked or inactivated, leading to necrotic cell death. Severe oxidative stress, which can be elicited by several pro-oxidants and hepatotoxic agents or drugs, leads to steady state concentrations of approx. $0.15 \mu \mathrm{M}$ for $\mathrm{H}_{2} \mathrm{O}_{2}$ and $0.25 \mu \mathrm{M}$ for total ROS. Less severe oxidative stress, not sufficient to irreversibly impair mitochondrial functions or to inactivate caspases, has been suggested to cause liver cell apoptosis ${ }^{20}$. It is reasonable to think that only mild levels of ROS may be generated in vivo during chronic liver disease. These relatively low levels of oxidative damage may sustain progression of fibrosis by the activation and phenotypical modulation of HSCs towards the so-called myofibroblast-like phenotype. Phenotypic responses of activated HSCs include proliferative status, synthesis and degradation/remodelling of extracellular matrix, chemotaxis, contractility, proinflammatory activity and retinoid loss. Oxidative stress may modulate collagen synthesis through the activation of $\mathrm{Na}^{+} / \mathrm{H}^{+}$exchanger and the increase in intracellular $\mathrm{pH}$. $\mathrm{H}_{2} \mathrm{O}_{2}$ and $\mathrm{O}_{2}^{-}$may induce the activation of collagen type I gene also by up-regulating cyclooxygenase 2 (COX2) and then, possibly, through the action of metabolites of arachidonic acid. $\mathrm{H}_{2} \mathrm{O}_{2}$ may further act as an intracellular signal mediator of the pro-fibrogenic action of tumour growth factor- $\beta$ (TGF- $\beta$ ) or of acetaldehyde and may up-regulate collagen type I gene by activation and binding of $\mathrm{p} 35 \mathrm{C} / \mathrm{EBP} 3$ protein to a specific region of the promoter of the collagen $\alpha 1$ (I) gene ${ }^{19}$.
Management of oxidative burst by extrinsic biologically active compounds

Different approaches are taken to modulate the oxidative burst by establishing an equilibrium shift in oxidant versus antioxidant status. Complete stoppage of the oxidative burst may defeat its purpose, i.e. dealing with injurious agents and the consequences of their presence, while attenuation could help to minimize damage to surrounding tissue.

The simplest approach, and one often used, is a diet supplemented with antioxidants. These are not limited to well-known antioxidants such as ascorbic acid or $\alpha$-tocopherol. Many natural compounds have the ability to scavenge ROS, thereby reducing oxidative stress directly, or they may offer an indirect protection by activating endogenous defense systems ${ }^{21}$. Induction of enzymes relevant in cell defense system, e.g. superoxide dismutase, catalase, glutathione peroxidase etc., seems conceivable because fully functional cells are more likely to respond as opposed to necrotic and damaged cells ${ }^{22}$.

Antioxidants isolated from plant and animal sources have been found within most natural substance classes, e. g. flavonoids, tannins, coumarins, xanthones or alkaloids. Flavonoids are by far the most comprehensively investigated natural products. Silymarin is a standardized extract of Silybum marianum and contains as the main active compounds the flavonoids silibinin, silychristine and silydianin. Silymarin suppresses expression of procollagen $\alpha 1$ (I) most likely via down-regulation of TGF- 11 mRNA in rats with biliary fibrosis. The antifibrotic effect of silymarin may result from its antioxidant and radical scavenging properties ${ }^{23}$. The flavonoid rutin is an efficient suppressor of oxygen radical overproduction by neutrophils of rheumatoid arthritis patients $^{24}$. Some results demonstrate that various flavonoids induce the cellular antioxidant system. The flavonoids quercetin, kaempferol and apigenin, as well as onion extract increase the $\gamma$-glutamylcysteine synthetase heavy subunit promoter activity in COS-1 cells. Quercetin and onion extract also increase the intracellular concentration of glutathione in these cells ${ }^{21}$.

The use of antioxidant resveratrol or specific inhibitors of the $\mathrm{Na}^{+} / \mathrm{H}^{+}$exchanger, amiloride and 5-N-ethyl-N-isopropylamiloride (EIPA), inhibit collagen synthesis by activated HSCs in vitro and in vivo and HSC proliferation. $\alpha$-Tocopherol suppresses release of TNF- $\alpha$ and IL- 6 in Kupffer cells, inhibits in vivo TGF- $\beta 1$ and MCP-1 expression and reduces the recruitment of leukocytes by inhibiting hydroxyalkenal-stimulated chemotaxis $^{19}$.

Baicalin, baicalein and wogonin, polyphenolic compounds isolated from the Chinese herb Huang Qui, inhibit LPS-induced NO production in RAW 264.7 macrophages through inhibition of inducible NO synthase (iNOS) gene expression, but not the activity of iNOS ${ }^{25}$.

A second approach, aimed more at the aftermath of inflammation rather than the process itself, is support of healthy tissue repair by e.g. phospholipid supply. 
Bulk doses of up to a gram of phospholipids may be administered but the efficacy of treatment is disputed due to preferred use of phospholipids as an energy source. Some beneficial effects though were reported for certain hepatic disorders ${ }^{26}$.

Affecting the oxidative burst by inhibiting the signalling cascade(s) leading to ROS production or NADPH oxidase is an area infrequently explored. Surprisingly so, since some features of signalling pathways are shared with the increased expression of pro-inflammatory cytokines to which much attention is paid (vide supra). It appears then that a modulation of respiratory burst offers the advantage of concomitant or consequent modulation of cytokine production.

NADPH oxidase, due to its role as the final step in superoxide formation, may be considered as the primary target for modulation. Highly intriguing data connecting NADPH oxidase and early ethanol-induced injury come from an in vivo study in rats ${ }^{27}$. It is in agreement with a hypothesis of the central role of NADPH oxidase in early liver injury.

An important crosspoint upstream from NADPH oxidase, and involved in many intracellular processes, is PKC. It is, in our view, the target of choice for the respiratory burst as well as cytokine production modulation. Indeed, this is an area under wide research scrutiny yielding many broad spectrum as well as specific inhibitors. Further upstream in the signalling pathway are calcium channels, phospholipase C, G-proteins, and cell receptors in that order (Fig. 2). Modulation of these, however, may have only limited effect or be relevant to a single stimulus.

Due to tentative dependence of the intracellular signals and multiprotein complexes assembly on cytoskeleton, agents exhibiting cytoskeleton disruption may be thought of as universal modulators. The downside is their toxicity because vital cell functions are frequently affected as well, resulting not only in oxidative burst inhibition but cell death. This is certainly the case for colchicine, a potent respiratory burst inhibitor, which causes both apoptosis and necrosis of neutrophil-like cells $^{28}$.

\section{CONCLUSION}

Kupffer cells stand at the turning point of the "vicious circle", a series of events which cause inflammation, especially chronic inflammation. Modulation of Kupffer cell functions, many of which are shared with neutrophils, may break the vicious circle thus delaying or preventing chronic inflammation and consequent liver fibrosis. It may even stop significant inflammation from occurring in the first place. Despite extensive data available on mechanisms involved in oxidative burst and cytokines expression, modulation of these has been no simple task to date. Future research will focus, as is the case with cancer cells, in finding an effective and specific modulator and, simultaneously, finding a way to deliver it solely to Kupffer cells.

\section{ACKNOWLEDGEMENTS}

Supported by grants IGUP 11501107 and MSM 1511 00003.

\section{REFERENCES}

1. Klaasen CD Casarett and Doull's Toxicology. $5^{\text {th }}$ edition, New York: McGraw-Hill, 1996.

2. Knolle P, Lohr H, Treichel U, Dienes HP, Lohse A, Schlaack J, Gerken G (1995) Parenchymal and nonparenchymal liver cells and their interaction in the local immune response. ZGastroenterol 33, 613-620.

3. Yata Y, Enosawa S, Suzuki S, Li XK, Tamura A, Kimura H, Takahara T, Watanabe A (1999) An improved method for the purification of stellate cells from rat liver with dichloromethylene diphosphate (CL2MDP). Methods Cell Sci 21, 19-24.

4. Rubin E, Farber JL Pathology. Philadelphia: J.P. Lippincott Company, 1988.

5. Klika E, Vacek Z, Dvořák M, Kapeller K, Histologie. Praha: Avicenum, 1986

6. Ham AW, Cormack DH, Histology. $8^{\text {th }}$ edition Toronto: J.P. Lippincott Company, 1972.

7. Decker K (1990) Biologically active products of stimulated liver macrophages (Kupffer cells). Eur J Biochem 192, 245-261.

8. Roitt IM, Delves PJ, Encyclopedia of immunology. Volume 2. London: Academic Press, 1992.

9. Roitt IM, Delves PJ, Encyclopedia of immunology. Volume 3. London: Academic Press, 1992.

10. Ramadori G, Armbrust T (2001) Cytokines in the liver. Eur J Gastroenterol Hepatol 13, 777-784.

11. Lichtman SN, Wang J, Lemasters JJ (1998) Lipopolysaccharidestimulated TNF-alpha release from cultured rat Kupffer cells: sequence of intracellular signaling pathways. J Leukoc Biol 64, 368-372.

12. Schultze RL, Gangopadhyay A, Cay O, Lazure D, Thomas P (1999) Tyrosine kinase activation in LPS stimulated rat Kupffer cells. Cell Biochem Biophys 30: 287-301.

13. Prabhakar U, Lipshutz D, Pullen M, Turchin H, Kassis S, Nambi P (1993) Protein kinase C regulates TNF-alpha production by human monocytes. Eur Cytokine Netw 4, 31-37.

14. Yui S, Mikami M, Mimaki Y, Sashida Y, Yamazaki M (2001) Inhibition effect of Amaryllidaceae alkaloids, lycorine and lycoricidinol on macrophage TNF-alpha production. Yakugaku Zasshi 121, 167-171.

15. Okamoto M, Ono M, Baba M (2001) Suppression of cytokine production and neural cell death by the anti-inflammatory alkaloid cepharanthine: a potential agent against HIV-1 encephalopathy. Biochem Pharmacol 62, 747-753.

16. Altindag ZZ, Werner-Felmayer G, Sahin G, Wachter H, Fuchs D (1997) Colchicine derivatives inhibit neopterin production in human peripheral blood mononuclear cells (PBMC). Clin Exp Immunol 107, 574-577.

17. Rao P, Falk LA, Dougherty SF, Sawada T, Pluznik DH (1997) Colchicine down-regulates lipopolysaccharide-induced granulocyte-macrophage colony-stimulating factor production in murine macrophages. J Immunol 159, 3531-3539.

18. Li N, Karin M (1999) Is NF-кB the sensor of oxidative stress? FASEB J 13, 1137-1143.

19. Parola M, Robino G (2001) Oxidative stress-related molecules and liver fibrosis. J Hepatol 35, 297-306.

20. Oliver JD, Roberts RA (2002) Receptor-mediated hepatocarcinogenesis: role of hepatocyte proliferation and apoptosis. Pharmacology \& Toxicology 91, 1-7.

21. Myhrstad MCW, Carlsen H, Nordstrom O, Blomhoff R, Moskaug JO (2002) Flavonoids increase the intracellular glutathione level 
by transactivation of the $\gamma$-glutamylcysteine synthetase catalytical subunit promoter. Free Radical Biol Med 32, 386-393.

22. Chiu H, Brittingham JA, Laskin DL (2002) Differential induction of heme oxygenase- 1 in macrophages and hepatocytes during acetaminophen-induced hepatotoxicity in the rat: effects of hemin and biliverdin. Toxicol Appl Pharmacol. 181, 106-115.

23. Jia J-D, Bauer M, Cho JJ, Ruehl M, Milani S, Boigk G, Riecken EO, Schuppan D (2001) Antifibrotic effect of silymarin in rat secondary biliary fibrosis is mediated by downregulation of procollagen $\alpha 1$ (I) and TIMP-1. J Hepatol 35, 392-398.

24. Ostrakhovitch EA, Afanas ev IB. (2001) Oxidative stress in rheumatoid arthritis leukocytes: suppression by rutin and other antioxidants and chelators. Biochem Pharmacol 62, 743-746.

25. Chen Y-C, Shen S-C, Chen L-G, Lee TJ-F, Yang L-L (2001) Wogonin, baicalin, and baicalein inhibition of inducible nitric oxide synthase and cyclooxygenase-2 gene expressions induced by nitric oxide synthase ihibitors and lipopolysaccharide. Biochem Pharmacol 61, 1417-1427.
26. Lata J, Dastych M Jr, Šenkyřík M, Husová M, Starý K (2001) [Protective effect of essential phospholipids on liver injury due to total parenteral nutrition] Vnitr Lek. 47, 599-603. Czech.

27. Kono H, Rusyn I, Uesugi T, Yamashina S, Connor HD, Dikalova A, Mason RP, Thurman RG (2001) Diphenyleneiodonium sulfate, an NADPH oxidase inhibitor, prevents early alcohol-induced liver injury in the rat. Am J Physiol Gastrointest Liver Physiol. 280, G1005-1012.

28. Vrba J, Modrianský M, Ulrichová J (2002) Effect of colchicine on oxidative burst in neutrophil-like cells. In: Timko J, Turňa J, and Pastorek J, editors. Zborník XVIII. Biochemický sjezd, Vysoké Tatry, Stará Lesná, SK, 10-13 September 2002. Bratislava: VEDA, 2002. p 329.

29. Kurosaka K, Watanabe N, Kobayashi Y (2001) Production of proinflammatory cytokines by resident tissue macrophages after phagocytosis of apoptotic cells. Cell Immunol 211, 1-7. 